\title{
MicroRNA-mediated Extracellular Matrix \\ Remodeling in Squamous Cell Carcinoma of the Oral Cavity
}

Gilberto Mendes Menderico Jr. ( $\square$ gilbertomendes.ccp@gmail.com )

FMUSP: Universidade de Sao Paulo Faculdade de Medicina https://orcid.org/0000-0001-7281-4044

Thérèse Rachell Theodoro

Faculdade de Medicina do ABC, Biochemistry Department

Fatima Solange Pasini

Centro de Investigação Translacional em Oncologia, Instituto do Câncer do Estado de São Paulo,

Faculdade de Medicina da Universidade de São Paulo

Marina de Menezes Ishikawa

Nursery School, Faculdade Israelita de Ciências da Saúde Albert Einsteins

Nayara Stephânie Sousa Santos

University of São Paulo Medical School

\section{Evandro Sobroza de Mello}

Pathology Department, University of São Paulo Medical School

\section{Maria Aparecida da Silva Pinhal}

Faculdade de Medicina da Fundacao do ABC: Faculdade de Medicina do ABC

\section{Raquel Ajub Moyses}

Head and Neck Surgery Department, University OF São Paulo Medical School

Marco Aurelio Vamondes Kulcsar

Head and Neck Surgery Department, University of São Paulo Medical School

\section{Rogério Aparecido Dedivitis}

Head and Neck Surgery Department, University of São Paulo Medical School

\section{Claudio Roberto Cernea}

Head and Neck Surgery Department, University of São Paulo Medical School

\section{Luiz Paulo Kowalski}

Head and Neck Surgery Department, University of São Paulo Medical School

\section{Leandro Luongo de Matos}

Head and Neck Surgery Department, University of São Paulo Medical School

Research article

Keywords: carcinoma, squamous cell, mouth, microRNAs, carcinogenesis 
Posted Date: October 29th, 2020

DOI: https://doi.org/10.21203/rs.3.rs-97307/v1

License: (9) This work is licensed under a Creative Commons Attribution 4.0 International License. Read Full License

Version of Record: A version of this preprint was published at Head \& Neck on April 8th, 2021. See the published version at https://doi.org/10.1002/hed.26686. 
MicroRNA-mediated extracellular matrix remodeling in squamous cell carcinoma of the oral cavity

\section{Gilberto Mendes Menderico Junior, PhD MD}

Head and Neck Surgery Department, Doctoral Degree, Anesthesiology, Surgical Sciences and Perioperative Medicine, University of Sao Paulo Medical School, Sao Paulo, Brazil

gilbertomendes.ccp@gmail.com

\section{Thérèse Rachell Theodoro, PhD}

Biochemistry Department, Faculdade de Medicina do ABC, Santo André, Brazil theodortr35@gmail.com

\section{Fatima Solange Pasini, PhD}

Centro de Investigação Translacional em Oncologia (CTO), Instituto do Cancer do Estado de Sao Paulo (ICESP), Hospital das Clinicas da Faculdade de Medicina da Universidade de Sao Paulo (HCFMUSP), Sao Paulo, Brazil

fatima.pasini@hc.fm.usp.br

\section{Marina de Menezes Ishikawa}

Nursery School, Faculdade Israelita de Ciências da Saúde Albert Einstein, Sao Paulo, Brazil

marina.ishikawa@uol.com.br

\section{Nayara Stephânie Sousa Santos}

Medical Student, University of Sao Paulo Medical School, Sao Paulo, Brazil nayara.sssantos@fm.usp.br

Evandro Sobroza de Mello, PhD MD 
Pathology Department, Instituto do Câncer do Estado de São Paulo (ICESP), Laboratório de Investigação Médica 14 (LIM14), University of Sao Paulo Medical School, Sao Paulo, Brazil

esobroza@gmail.com

\section{Maria Aparecida da Silva Pinhal, PhD}

Biochemistry Department, Faculdade de Medicina do ABC, Santo André, Brazil maspinhal@yahoo.com.br

\section{Raquel Ajub Moyses, MD PhD MPH}

Head and Neck Surgery Department, Laboratório de Investigação Médica 28 (LIM28), University of Sao Paulo Medical School, Sao Paulo, Brazil ramccp@gmail.com

\section{Marco Aurelio Vamondes Kulcsar, MD PhD}

Head and Neck Surgery Department, Instituto do Câncer do Estado de São Paulo (ICESP), University of Sao Paulo Medical School, Sao Paulo, Brazil

kulcsar@uol.com.br

\section{Rogério Aparecido Dedivitis, MD PhD}

Head and Neck Surgery Department, University of Sao Paulo Medical School, Sao Paulo, Brazil

dedivitis.hns@uol.com.br

\section{Claudio Roberto Cernea, MD PhD}

Head and Neck Surgery Department, University of Sao Paulo Medical School, Sao Paulo, Brazil

cerneamd@gmail.com

\section{Luiz Paulo Kowalski, MD PhD}


Head and Neck Surgery Department, University of Sao Paulo Medical School, Sao Paulo, Brazil

lp_kowalski@uol.com.br

\section{Leandro Luongo de Matos, MD PhD}

Head and Neck Surgery Department, Instituto do Câncer do Estado de São Paulo (ICESP), Laboratório de Investigação Médica 28 (LIM28), University of Sao Paulo Medical School, Sao Paulo, Brazil

lematos@gmail.com

Study developed at Head and Neck Surgery Discipline, Surgery Department, University of Sao Paulo Medical School, Sao Paulo, SP, Brazil

Corresponding Authors: Gilberto Mendes Menderico Junior. Enéas de Carvalho Aguiar avenue, 255, 8th floor, room 8174, 05403-000, São Paulo, SP, Brazil. Fone: +55 11 26616425. Email: 1.matos@fm.usp.br; gilbertomendes.ccp@gmail.com 
The authors of the study "MicroRNA-mediated extracellular matrix remodeling in squamous cell carcinoma of the oral cavity" declare that it was approved by the Institutional Review Board of the University of São Paulo Medical School under protocol number 228/14 (CAAE: 32884214.5.0000.0065). Informed consent was obtained from all participants and/or legal guardians during medical visits, in accordance with Ethical Principals for Medical Research Involving Human Subjects (World Medical Association Declaration of Helsinki). All experiments were performed in accordance to manufactures' instructions as properly detailed in this session, in accordance with Brazilian Government Regulatory Agency (ANVISA - Agência Nacional de Vigilância Sanitária).

Availability of data and materials: The datasets used and/or analysed during the current study are available from the corresponding author on reasonable request.

Competing Interest: The authors declare that they have no competing interests

Funding: Fundação de Amparo à Pesquisa do Estado de São Paulo (FAPESP - process 2016/01740-6)

\section{Authors contributions:}

G.M.M.J and L.L.M. conceived the experiment(s), G.M.M.J, T.R.T, M.M.I., N.S.S.S., M.A.S.P. and F.S.P conducted the experiment(s), L.L.M, E.S.M., M.A.V.K., R.A.M and F.S.P. analysed the results and statistics. R.A.D, G.M.M.J prepared the manuscript. L.P.K and C.R.C. reviewed the manuscript. 


\section{Abstract}

Background: It has been demonstrated that for oral cavity squamous cell carcinoma (SCC) as tumor thickness increases, there are lower rates of survival. Several molecular factors have been studied to determine this invasive behavior, including microRNAs, that play a role in the tumor microenvironment. Methods: The aim of this study is evaluate the role of extracellular matrix remodeling, as well as the involvement of microRNAs, in the process of oral cavity SCC carcinogenesis. This was a retrospective study with patients operated on for SCC of the oral cavity, in addition to a group of oral mucosa samples from paired patients. RNA extraction was performed, followed by complementary DNA amplification of microRNAs related to adhesion, migration, cell proliferation, apoptosis, and constituents of the extracellular matrix. We also performed immunohistochemical reactions for markers involved in the same biological processes. Results: High expression of miR-21-5p and miR-106-5p and low expression of miR320a and miR-222-3p were predictors of malignancies and mir21-5p, individually, showed the best differentiation between the groups $($ AUC $=0.972)$. Regarding the immunohistochemical markers, there was greater expression of p53, EGFR, metalloproteinase-2 (MMP-2), laminin beta, Ki-67 and CD34 in the tumor cells than in the healthy mucosa. Furthermore, increased expression of MMP-2, metalloproteinase-9 (MMP-9), laminin alpha and laminin beta in tumor-related fibroblasts and lower continuity of type IV collagen in the basement membrane were observed. Conclusions: These results demonstrate the biological effects of microRNAs on the carcinogenesis of SCC of the oral cavity as well as the intense modification of the tumor microenvironment.

Keywords: carcinoma, squamous cell; mouth; microRNAs; carcinogenesis 


\section{Introduction}

Malignant neoplasms of the oral cavity are a major public health problem, given their increased incidence in the world and their unchanged mortality rate, despite advances in therapeutic modalities (1-3). The most common histological type is squamous cell carcinoma (SCC), which accounts for more than $90 \%$ of cases and whose main risk factor is smoking and its association with alcohol abuse (4-7). The presence of lymph node metastases is one of the main factors contributing to worsening prognoses of patients with SCC of the oral cavity, and the greater the infiltration of the neoplasia is, as measured by tumor thickness or depth of invasion, the greater the contact of neoplastic cells with blood and lymphatic vessels, with consequent development of metastases (813).

Among the factors that determine the highest invasion rate of a neoplasm is its interaction with the basement membrane and the extracellular matrix (ECM), making the environment more conducive to remodeled cell growth (14-17). Thought in the past as only a support structure composed of fibrillar proteins (collagen and elastin), proteoglycans and structural glycoproteins, today, the ECM has been shown to be extremely important for tumor progression, due to the degradation of its components and angiogenesis, in addition to its role in proliferation, neoplasm migration and apoptosis $(18-20)$.

Several genetic and epigenetic factors, including microRNAs (miRNAs), have been implicated in the interaction between neoplasias and the ECM, thus allowing for its remodeling and tumor invasion (21).

miRNAs are small non-coding RNA molecules composed of 19 to 25 nucleotides that act by binding to the untranslated 3' region of messenger RNA (mRNA), resulting in degradation or translation inhibition. miRNAs act by negatively regulating 
gene expression at the posttranscription level (22). Previous studies have shown that the expression of miRNAs is dysregulated in several types of cancer, as more than $50 \%$ of the genes that regulate miRNA expression are found in fragile sites of chromosomes, which are susceptible to deletions or rearrangements. This dysregulation of miRNA expression may influence cell differentiation and proliferation and, therefore, miRNAs can act by themselves as oncogenes or tumor suppressor genes (23-26).

Although several studies are being developed to determine the influence of miRNAs on the progression of SCC of the oral cavity, it is still difficult to identify biomarkers with good accuracy for predicting malignancy. The aim of this study is to evaluate the role of extracellular matrix remodeling, as well as the involvement of microRNAs, in the process of oral cavity SCC carcinogenesis. 


\section{Methods}

This study was approved by the Institutional Review Board under protocol number (CAAE: 32884214.5.0000.0065). The informed consent was obtained from all the patients, except from those who have of lost ambulatory follow-up and the dead, which was dispensed as it is a retrospective study, with analysis of paraffined material.

A total of 26 cases were selected, and remnant paraffin blocks containing SCC of the oral cavity samples obtained from patients who underwent primary curative resection from December 2010 to October 2015 in the Head and Neck Surgery Service of the Cancer Institute of the State of São Paulo (Instituto do Câncer do Estado de São Paulo - ICESP), Sao Paulo, Brazil, were used. Tissues of oral mucosa preserved in paraffin blocks obtained from 11 patients who underwent surgeries due to benign oral cavity diseases were also included, constituting the control group.

Demographic, clinical and anatomopathological data were obtained from electronic medical records.

Tumor thickness measurements were performed using slides stained with hematoxylin and eosin by an independent pathologist. Representative areas with SCC were selected for tissue microarray (TMA) construction and immunohistochemical analysis of the chosen patient blocks.

\section{MicroRNA detection technique}

The level of microRNA expression was assessed by means of quantitative reverse transcription polymerase chain reaction (RT-qPCR). The RNA contained in 10 $5 \mu \mathrm{m}$ sections of paraffin-embedded tissue was extracted using a MagMAX ${ }^{\circledR}$ FFPE RNA Ultra Kit (Applied Biosystems ${ }^{\circledR}$, Foster City, Ca, USA) according to the manufacturer's 
protocol. RT-qPCR was performed using the TaqMan ${ }^{\circledR}$ Low Density Array (TLDA; Thermo Fisher Scientific ${ }^{\circledR}$, Waltham, Ma, USA) system according to the manufacturer's protocol. RNA was reverse transcribed and polyadenylated according to the provided protocol, generating cDNA. All amplifications were performed in triplicate, and the cycle threshold (CT) values were determined with the Cloud platform (Thermo Fisher Scientific $^{\circledR}$, Waltham, Ma, USA). miRNAs with CT values > 38 were considered indeterminate and discarded. Only miRNAs detected in at least $68 \%$ of the samples were considered for statistical analysis. Normalization between samples was performed using the Quantil method using Expander software, and miR-let-7i was used to normalize the expression values of the remaining miRNAs. A list of primers for the miRNAs studied is provided in Table 5

Table 5. Primer sequences used in the microRNA research.

\begin{tabular}{|lc|}
\hline microRNA & Primer sequence \\
cel-mir-39-3p & UCACCGGGUGUAAAUCAGCUUG \\
hsa-let-7b-5p & UGAGGUAGUAGGUUGUGUGGUU \\
hsa-let-7d-5p & AGAGGUAGUAGGUUGCAUAGUU \\
hsa-let-7i-5p & UGAGGUAGUAGUUUGUGCUGUU \\
hsa-mir-1-3p & UGGAAUGUAAAGAAGUAUGUAU \\
hsa-mir-106b-5p & UAAAGUGCUGACAGUGCAGAU \\
hsa-mir-107 & AGCAGCAUUGUACAGGGCUAUCA \\
hsa-mir-10a-5p & UACCCUGUAGAUCCGAAUUUGUG \\
hsa-mir-10b-5p & UACCCUGUAGAACCGAAUUUGUG \\
hsa-mir-124-3p & UAAGGCACGCGGUGAAUGCC \\
hsa-mir-1258 & AGUUAGGAUUAGGUCGUGGAA \\
hsa-mir-125a-3p & ACAGGUGAGGUUCUUGGGAGCC \\
hsa-mir-125b-5p & UCCCUGAGACCCUAACUUGUGA \\
hsa-mir-126-3p & UCGUACCGUGAGUAAUAAUGCG \\
hsa-mir-132-3p & UAACAGUCUACAGCCAUGGUCG \\
hsa-mir-133a-3p & UUUGGUCCCCUUCAACCAGCUG \\
hsa-mir-137 & UUAUUGCUUAAGAAUACGCGUAG \\
hsa-mir-138-5p & AGCUGGUGUUGUGAAUCAGGCCG \\
hsa-mir-143-3p & UGAGAUGAAGCACUGUAGCUC \\
hsa-mir-147b & GUGUGCGGAAAUGCUUCUGCUA \\
hsa-mir-155-5p & UUAAUGCUAAUCGUGAUAGGGGU \\
hsa-mir-16-5p & UAGCAGCACGUAAAUAUUGGCG \\
hsa-mir-17-5p & CAAAGUGCUUACAGUGCAGGUAG \\
hsa-mir-184 & UGGACGGAGAACUGAUAAGGGU \\
hsa-mir-192-5p & CUGACCUAUGAAUUGACAGCC \\
hsa-mir-200a-3p & UAACACUGUCUGGUAACGAUGU \\
hsa-mir-200b-3p & UAAUACUGCCUGGUAAUGAUGA \\
hsa-mir-200c-3p & UAAUACUGCCGGGUAAUGAUGGA \\
hsa-mir-203a-3p & GUGAAAUGUUUAGGACCACUAG \\
hsa-mir-204-5p & UUCCCUUUGUCAUCCUAUGCCU \\
hsa-mir-205-5p & UCCUUCAUUCCACCGGAGUCUG \\
hsa-mir-21-5p & UAGCUUAUCAGACUGAUGUUGA \\
hsa-mir-211-5p & UUCCCUUUGUCAUCCUUCGCCU \\
hsa-mir-212-3p & UAACAGUCUCCAGUCACGGCC \\
hsa-mir-218-5p & UUGUGCUUGAUCUAACCAUGU \\
hsa-mir-219a-5p & UGAUUGUCCAAACGCAAUUCU \\
&
\end{tabular}




\begin{tabular}{lc} 
hsa-mir-221-3p & AGCUACAUUGUCUGCUGGGUUUC \\
hsa-mir-222-3p & AGCUACAUCUGGCUACUGGGU \\
hsa-mir-223-3p & UGUCAGUUUGUCAAAUACCCCA \\
hsa-mir-27a-3p & UUCACAGUGGCUAAGUUCCGC \\
hsa-mir-29a-3p & UAGCACCAUCUGAAAUCGGUUA \\
hsa-mir-29b-3p & UAGCACCAUUUGAAAUCAGUGUU \\
hsa-mir-29c-3p & UAGCACCAUUUGAAAUCGGUUA \\
hsa-mir-30a-5p & UGUAAACAUCCUCGACUGGAAG \\
hsa-mir-31-5p & AGGCAAGAUGCUGGCAUAGCU \\
hsa-mir-320a & AAAAGCUGGGUUGAGAGGGCGA \\
hsa-mir-330-3p & GCAAAGCACACGGCCUGCAGAGA \\
hsa-mir-335-5p & UCAAGAGCAAUAACGAAAAAUGU \\
hsa-mir-340-5p & UUAUAAAGCAAUGAGACUGAUU \\
hsa-mir-34a-5p & UGGCAGUGUCUUAGCUGGUUGU \\
hsa-mir-34c-5p & AGGCAGUGUAGUUAGCUGAUUGC \\
hsa-mir-373-3p & GAAGUGCUUCGAUUUUGGGGUGU \\
hsa-mir-375 & UUUGUUCGUUCGGCUCGCGUGA \\
hsa-mir-376c-3p & AACAUAGAGGAAAUUCCACGU \\
hsa-mir-378a-3p & ACUGGACUUGGAGUCAGAAGGC \\
hsa-mir-485-5p & AGAGGCUGGCCGUGAUGAAUUC \\
hsa-mir-489-3p & GUGACAUCACAUAUACGGCAGC \\
hsa-mir-494-3p & UGAAACAUACACGGGAAACCUC \\
hsa-miR-498 & UUUCAAGCCAGGGGGCGUUUUUC \\
hsa-mir-518d-5p & CUCUAGAGGGAAGCACUUUCUG \\
hsa-mir-9-5p & UCUUUGGUUAUCUAGCUGUAUGA \\
hsa-miR-940 & AAGGCAGGGCCCCCGCUCCCC \\
hsa-mir-96-5p & UUUGGCACUAGCACAUUUUUGCU \\
hsa-mir-99a-5p & AACCCGUAGAUCCGAUCUUGUG \\
& \\
\hline
\end{tabular}

Immunohistochemical analysis

Immunohistochemical detection of the various proteins was performed on slides obtained from the TMA blocks. The following antibodies and dilutions were used: p16 (clone E6H4, pH 9.3, ready for use, Ventana, USA), p53 (clone DO-7, pH 9.3, ready for use, Ventana, USA), epidermal growth factor (EGFR, EGFR pharmDxTM kit., pH 9.3, Dako Byogen, USA), cluster of differentiation 34 (CD34, clone QBEnd-10, pH 9.3, ready for use, Ventana, USA), BCL-2 protein (clone SP66, pH 9.3, ready for use, Ventana, USA), type IV collagen (clone CIV22, pH 9.3, ready for use, Ventana, USA), Ki-67 antigen (clone 30-9, 1: 100 dilution, Ph 9.3, Ventana, USA), MMP-2 (clone sc-13595, pH 6.3, 1:50 dilution, Santa Cruz Biotechnology ${ }^{\circledR}$, Dallas, Texas, USA), MMP-9 (clone sc6840, pH 6.3, 1:100 dilution, Santa Cruz Biotechnology ${ }^{\circledR}$, Dallas, Texas, USA), heparanase (HPA1 H80, clone sc25825, pH 6.3, 1:100 dilution, Santa Cruz Biotechnology ${ }^{\circledR}$, Dallas, Texas, USA), HPSE2 (C-17-sc-14900 HPA2 (goat pAb), pH 9.3, 
1: 200 dilution, Santa Cruz Biotechnology ${ }^{\circledR}$, Dallas, Texas, USA), laminin alpha (goat polyclonal antibody (M-20) sc-6017, pH 9.3, 1: 100 dilution, Santa Cruz Biotechnology ${ }^{\circledR}$, Dallas, Texas, USA), laminin beta (goat polyclonal antibody (C-19) sc-6018, pH 9.3, 1:100 dilution, Santa Cruz Biotechnology ${ }^{\circledR}$, Dallas, Texas, USA), syndecan 1 (B-A38 mouse monoclonal antibody, ready for use, $\mathrm{pH}$ 9.3, Cell Marque Corporation - USA), syndecan 4 (H-140: sc-15350, 1:100 dilution, pH 9.3, Santa Cruz Biotechnology ${ }^{\circledR}$, Dallas, Texas, USA) and decorin (N-15: sc-22613 goat polyclonal antibody, dilution 1:100, pH 9.3, Santa Cruz Biotechnology ${ }^{\circledR}$, Dallas, Texas, USA). The sections were deparaffinized and rehydrated and the antigen was recovered in citrate buffer solution $\mathrm{pH}$ 6.0 (PMB1125 - Spring Bioscience ${ }^{\circledR}-$ Switzerland) or Tris-EDTA buffer solution, pH 9.0 (PMB4125- Spring Bioscience ${ }^{\circledR}$ - Switzerland) in a steamer for 35 minutes. Blocking of endogenous peroxidase was then performed using $6 \% \mathrm{H}_{2} \mathrm{O}_{2}$, and the sections were incubated with the primary antibody at $37{ }^{\circ} \mathrm{C}$ for 30 minutes, followed by incubation at 4 ${ }^{\circ} \mathrm{C}$ overnight (18 hours). Next, incubation was performed with a high-sensitivity amplification system based on the avidin-biotin-free polymer NovoLink (Polimer Leica Biosystems, Newcastle Ltd, UK), and primary antibodies produced in goats were incubated with anti-goat IgG (Immpress ${ }^{\circledR}$ HRP Reagent Kit - Peroxidase cat. No. MP7405, Sigma Aldrich, San Luis, Missouri, USA). The reaction was developed with chromogenic substrate solution containing diaminobenzidine (Sigma Aldrich, San Luis, Missouri, USA) and counterstained with Meyer's hematoxylin.

The evaluation of the immunohistochemical expression of p16, p53, bcl-2, collagen IV, EGFR, MMP-2, MMP-9, heparanase, HSPE2, laminin alpha, laminin beta, syndecan 1, syndecan 4, and decorin in neoplastic cells and for MMP-2, MMP-9, laminin alpha and laminin beta in fibroblasts was performed under light microscopy (Nikon eclipse e200 ${ }^{\circledR}$ microscope, Tokyo, Japan) and using Pannoramic Viewer ${ }^{\circledR}$ software 
(3DHistech Ltd, Budapest, Hungary) using a semiquantitative method. The grading for positively stained cells was as follows: 0 (no cells stained), > $0-10 \%, 10-25 \%, 25$ $50 \%, 50-75 \%$ and $>75 \%$.

Regarding the Ki-67 antigen, its evaluation was quantitatively performed with the count of the first 500 tumor cells detected, counting from the first section evaluated, excluding areas of inflammation, fibrosis, necrosis and poor fixation. After counting 500 cells, the percentage of stained cells was obtained, regardless of the staining intensity (27).

Immunoreactivity to CD34 and VEGFR3 enabled us to calculate the microvessel density (MVD), obtained by the arithmetic mean of the number of vessels present in four sections from the same patient. All stained endothelial cells and cell clusters were counted as microvessels. For analysis, counts were performed at 400x magnification (28).

\section{Statistical analysis}

The values obtained by the study of each continuous parametric distribution variable were organized and described using the mean and standard deviation (SD) or standard error (SE). For categorical variables, absolute and relative frequencies were used. The cutoff values for quantitative variables were determined by receiver operating characteristic (ROC) curve analysis, and those for qualitative variables were determined by category associations. The Mann-Whitney test was used to compare quantitative variables between two sample populations. Additionally, variables with P-value $<0.10$ in the univariate analysis were subjected to multivariate analyses, which were performed through linear regression models, for the selection of independent quantitative variables. Frequency comparisons were performed using Fisher's exact test. Accuracy estimates (area under the ROC curve - AUC), in addition to sensitivity and specificity, were also 
determined by ROC curve analysis (positive Z-score values for expression were used in cases of overexpressed microRNAs, and the negative Z-score values for expression were used in cases of underexpressed microRNAs). Statistical software SPSS $^{\circledR}$ version 26.0 (SPSS $^{\circledR}$ Inc; Illinois, USA) was used for all analyses. The $\mathrm{MeV}$ program (MultiExperiment Viewer version 4.5.0; available at http://mev.tm4.org) was used to obtain the heatmap plots (29).

\section{Results}

\section{Group characteristics}

Twenty-six patients diagnosed with SCC of the oral cavity were included in the study. Of these, 18 were men (69.2\%), with a mean age of 62.4 years (SD of 8.9 years). Eleven specimens of healthy mucosa obtained from surgeries involving benign lesions of the oral cavity were included, composing the "control" group of the study. Of these, nine were men $(81.8 \%)$, with a mean age of 54 years (SD of 9.2 years), and six were still smokers $(54.5 \%)$. At the time of the anatomopathological analysis of the surgical excision product, 15 patients $(57 \%)$ had lymph node metastasis, 11 with extracapsular extension. The mean follow-up period was 21 months. There were seven deaths $(26.9 \%)$ due to the disease during the study period. The demographic and clinical variables of the study are shown in Table 1.

Table 1. Demographic, clinical and anatomopathological characteristics of patients with squamous cell carcinoma of the oral cavity included in the study.

\begin{tabular}{lc}
\hline \multicolumn{1}{c}{ Variable } & Patients with SCC of the Oral Cavity \\
\hline Male sex & $18(69.23 \%)$ \\
Age & $62.38 \pm 8.9$ years \\
Primary site & \\
Retromolar area & $2(7.6 \%)$ \\
Gingival ridge & $3(11.5 \%)$ \\
Tongue & $13(50 \%)$ \\
Mouth floor & $4(15.3 \%)$
\end{tabular}




$\begin{array}{lc}\text { Jugal mucosa } & 1(3.8 \%) \\ \text { Hard palate } & 3(11.5 \%) \\ \text { Smoking } & 20(76.9 \%) \\ \text { Alcohol abuse } & 15(57.7 \%) \\ \text { Degree of differentiation } & \\ \text { Well } & 8(30.7 \%) \\ \text { Moderate } & 16(61.5 \%) \\ \text { Poor } & 2(7.7 \%) \\ \text { Perineural invasion } & 9(34.6 \%) \\ \text { Angiolymphatic invasion } & 3(11.5 \%) \\ \text { Lymph node metastasis } & 15(57 . \%) \\ \text { Extracapsular spread } & 11(42.3 \%) \\ \text { Adjuvant radiotherapy } & 16(61.5 \%) \\ \text { Adjuvant chemotherapy } & 6(23 \%) \\ \text { Locoregional recurrence } & 2(7.7 \%) \\ \text { Distant metastasis } & 2(7.7 \%) \\ \text { Death } & 7(26.9 \%)\end{array}$

Analysis of the microRNA profile for the differentiation of normal mucosa from SCC of the oral cavity

Lower expression of miR-10b-5p, miR-221-3p, miR-222-3p, miR-320a, miR485-5p and miR-99a-5p and higher expression of miR-106b-5p, miR-21-5p, miR-223-3p and miR-34a-5p were observed in the tumor tissue compared to the healthy mucosa in the univariate analysis. The complete results are provided in Table 2.

Table 2. Univariate analysis of microRNAs differentially expressed between normal mucosa and tumor specimens, normalized by miR-let-7i.

\begin{tabular}{lccccc}
\hline microRNA & \multicolumn{2}{c}{$\begin{array}{c}\text { NORMAL } \\
\text { MUCOSA }\end{array}$} & \multicolumn{2}{c}{ TUMOR } & $\begin{array}{c}\text { P-value } \\
\text { (Mann- } \\
\text { Whitney) }\end{array}$ \\
\hline miR-let7b-5p & -1.427 & 0.275 & -1.824 & 0.213 & 0.332 \\
miR-let7d-5p & -4.606 & 0.366 & -5.213 & 0.198 & 0.192 \\
miR-1-3p & -1.779 & 0.399 & -0.212 & 0.275 & 0.120 \\
miR-106b-5p & -4.496 & 0.395 & -3.219 & 0.295 & $\mathbf{0 . 0 3 0}$ \\
miR-10b-5p & -2.908 & 0.270 & -4.216 & 0.195 & $<\mathbf{0 . 0 0 1}$ \\
miR-1258 & -0.745 & 0.206 & -1.056 & 0.156 & 0.228 \\
miR-125b-5p & -0.190 & 0.226 & -0.648 & 0.174 & 0.132 \\
miR-126-3p & -0.955 & 0.254 & -1.143 & 0.201 & 0.614 \\
miR-133a-3p & -2.280 & 0.518 & -2.509 & 0.726 & 0.485 \\
miR-143-3p & -1.444 & 0.347 & -0.616 & 0.144 & $\mathbf{0 . 0 2 8}$
\end{tabular}




\begin{tabular}{llllll} 
miR-155-5p & -6.445 & 0.658 & -6.598 & 0.364 & 0.949 \\
miR-16-5p & -4.411 & 0.397 & -4.018 & 0.226 & 0.454 \\
miR-17-5p & -4.737 & 0.416 & -5.141 & 0.342 & 0.562 \\
miR-184 & -3.706 & 0.864 & -3.533 & 0.424 & 0.864 \\
miR-192-5p & -4.221 & 1.012 & -4.731 & 0.555 & 0.828 \\
miR-200a-3p & -2.763 & 0.189 & -2.678 & 0.243 & 0.961 \\
miR-200b-3p & -0.822 & 0.311 & -0.337 & 0.279 & 0.256 \\
miR-200c-3p & -1.836 & 0.173 & -1.524 & 0.217 & 0.460 \\
miR-203a-3p & -2.535 & 0.383 & -3.227 & 0.275 & 0.271 \\
miR-205-5p & 0.197 & 0.407 & -0.091 & 0.213 & 0.316 \\
miR-21-5p & -2.010 & 0.260 & 0.229 & 0.176 & $<\mathbf{0 . 0 0 1}$ \\
miR-221-3p & -1.582 & 0.296 & -2.542 & 0.230 & $\mathbf{0 . 0 1 2}$ \\
miR-222-3p & -4.557 & 0.238 & -5.913 & 0.316 & $\mathbf{0 . 0 1 3}$ \\
miR-223-3p & -5.383 & 0.326 & -3.959 & 0.206 & $\mathbf{0 . 0 0 5}$ \\
miR-27a-3p & -2.493 & 0.350 & -1.786 & 0.183 & 0.097 \\
miR-29a-3p & -3.036 & 0.412 & -2.791 & 0.178 & 0.653 \\
miR-29b-3p & -3.261 & 0.315 & -2.501 & 0.188 & 0.109 \\
miR-29c-3p & -2.961 & 0.353 & -2.365 & 0.155 & 0.130 \\
miR-30a-5p & -0.904 & 0.358 & -0.637 & 0.304 & 0.553 \\
miR-31-5p & -4.736 & 0.578 & -4.627 & 0.292 & 0.701 \\
miR-320a & -0.538 & 0.318 & -1.794 & 0.294 & $\mathbf{0 . 0 1 6}$ \\
miR-34a-5p & -3.005 & 0.301 & -2.162 & 0.162 & $\mathbf{0 . 0 3 3}$ \\
miR-373-3p & -6.741 & 0.690 & -7.719 & 0.393 & 0.219 \\
miR-375 & -1.246 & 0.381 & -0.662 & 0.203 & 0.196 \\
miR-376c-3p & -6.667 & 0.297 & -6.685 & 0.485 & 0.668 \\
miR-378a-3p & -2.452 & 0.301 & -2.950 & 0.452 & 0.876 \\
miR-485-5p & -2.970 & 0.554 & -5.621 & 0.448 & $\mathbf{0 . 0 0 8}$ \\
miR-9-5p & -2.377 & 0.936 & -2.015 & 0.301 & 0.781 \\
miR-99a-5p & -1.786 & 0.293 & -2.738 & 0.191 & $\mathbf{0 . 0 1 3}$ \\
\hline
\end{tabular}

Legend: $\mathrm{SE}=$ standard-error 
The multivariate analysis (linear regression) showed that among microRNAs identified as differentially expressed, high expression of miR-21-5p (Beta=0.131; CI95\%: $0.070-0.191 ; \mathrm{P}<0.001)$ and $\mathrm{miR}-106-5 \mathrm{p}(\mathrm{Beta}=0.149 ; \mathrm{CI} 95 \%: 0.094-0.203 ; \mathrm{P}<0.001)$ and low expression of miR-320a (Beta=-0.092; CI95\%: $-0.139--0.045 ; \mathrm{P}=0.001)$ and miR-222-3p (Beta=-0.107; CI95\%: $-0.158--0.056 ; \mathrm{P}=0.001)$ were predictors of malignancies. Furthermore, the association of these four microRNAs showed good differentiation between the samples (linear regression model; $\mathrm{R}^{2}=0.926, \mathrm{SE}=0.119 ; \mathrm{p}$ $=0.001)$, as demonstrated in the heatmap provided in Figure 1.

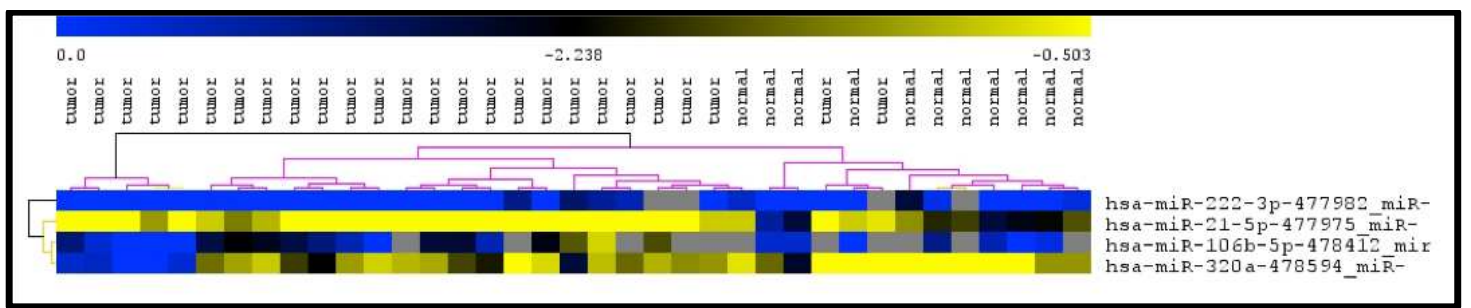

Figure 1. Heatmap showing the differentially expressed microRNAs and hierarchical cluster analysis of the samples for the differentiation of tumor specimens from healthy mucosa, considering only the microRNAs differentially expressed in the multivariate analysis (miR -21-5p, miR320a, miR-106b-5p and miR-222-3p). Yellow areas represent samples with lower expression, and blue areas represent samples with higher microRNA expression.

Estimating the accuracy for the differential diagnosis of tumor tissue from healthy mucosa, individually, miR-21-5p (AUC $=0.972 ; 95 \%$ CI: $0.911-1.000)$ exhibited the best statistical performance, followed very similarly by miR- 222-3p (AUC $=0.798 ; 95 \%$ CI: $0.620-0.975), \operatorname{miR}-320 \mathrm{a}(\mathrm{AUC}=0.786 ; 95 \% \mathrm{CI}: 0.570-1.030)$ and $\mathrm{miR}-106-5 \mathrm{p}$ $(\mathrm{AUC}=0.770,95 \% \mathrm{CI}: 0.586-0.954)$, which were studied as continuous variables. The curves are shown in Figure 2. 


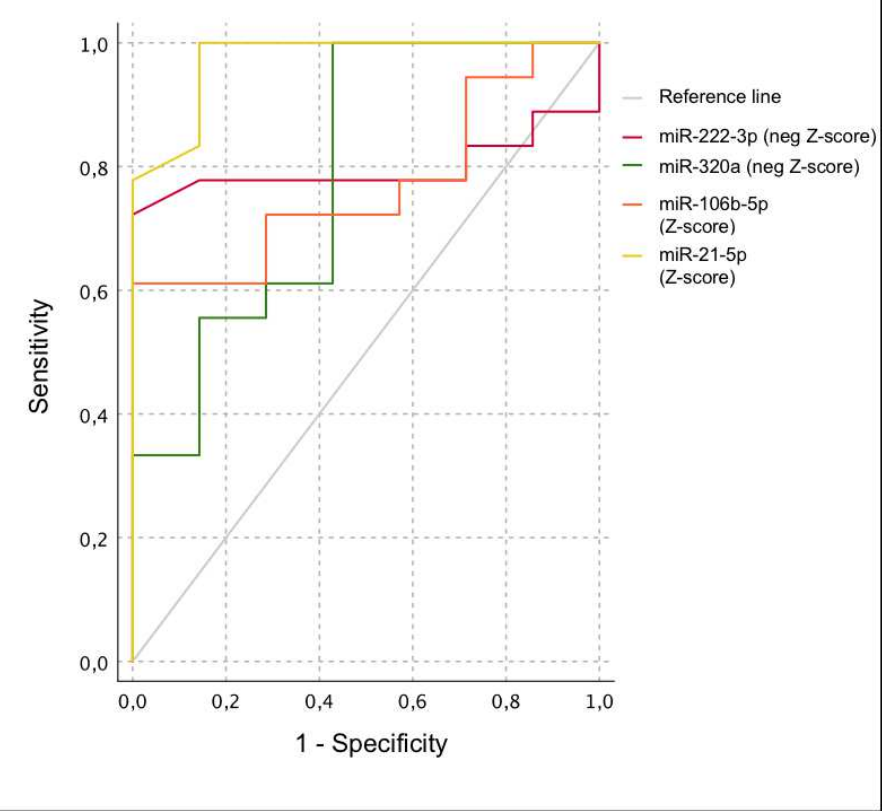

Figure 2. ROC (receiver operating characteristic) curve demonstrating the accuracy of miRNAs 21-5p, 106b-5p, 222-3p and 320a for the differential diagnosis of squamous cell carcinoma of the oral cavity from healthy mucosa.

Analysis of the expression of immunohistochemical markers in the normal mucosa and SCC of the oral cavity

Regarding the immunohistochemical markers, there was greater expression of p53, EGFR, metalloproteinase-2 (MMP-2), laminin beta, Ki-67 and CD34 in the tumor cells than in the healthy mucosa. Furthermore, increased expression of MMP-2, metalloproteinase-9 (MMP-9), laminin alpha and laminin beta in tumor-associated fibroblasts and lower continuity of type IV collagen in the basement membrane were observed. The complete data are provided in Table 3. These results demonstrate higher rates of cell proliferation, neoangiogenesis and extracellular matrix degradation in tumor specimens, including tumor-associated fibroblasts.

Table 3. Differential expression of stratified immunohistochemical markers for the differentiation of tumor specimens from healthy mucosa.

\begin{tabular}{cccc}
\hline GROUP/MARKER & IMMUNOEXPRESSION & P-value (Fisher exact test) \\
\hline BCL2 & $\leq 10 \%$ & $>10 \%$ &
\end{tabular}




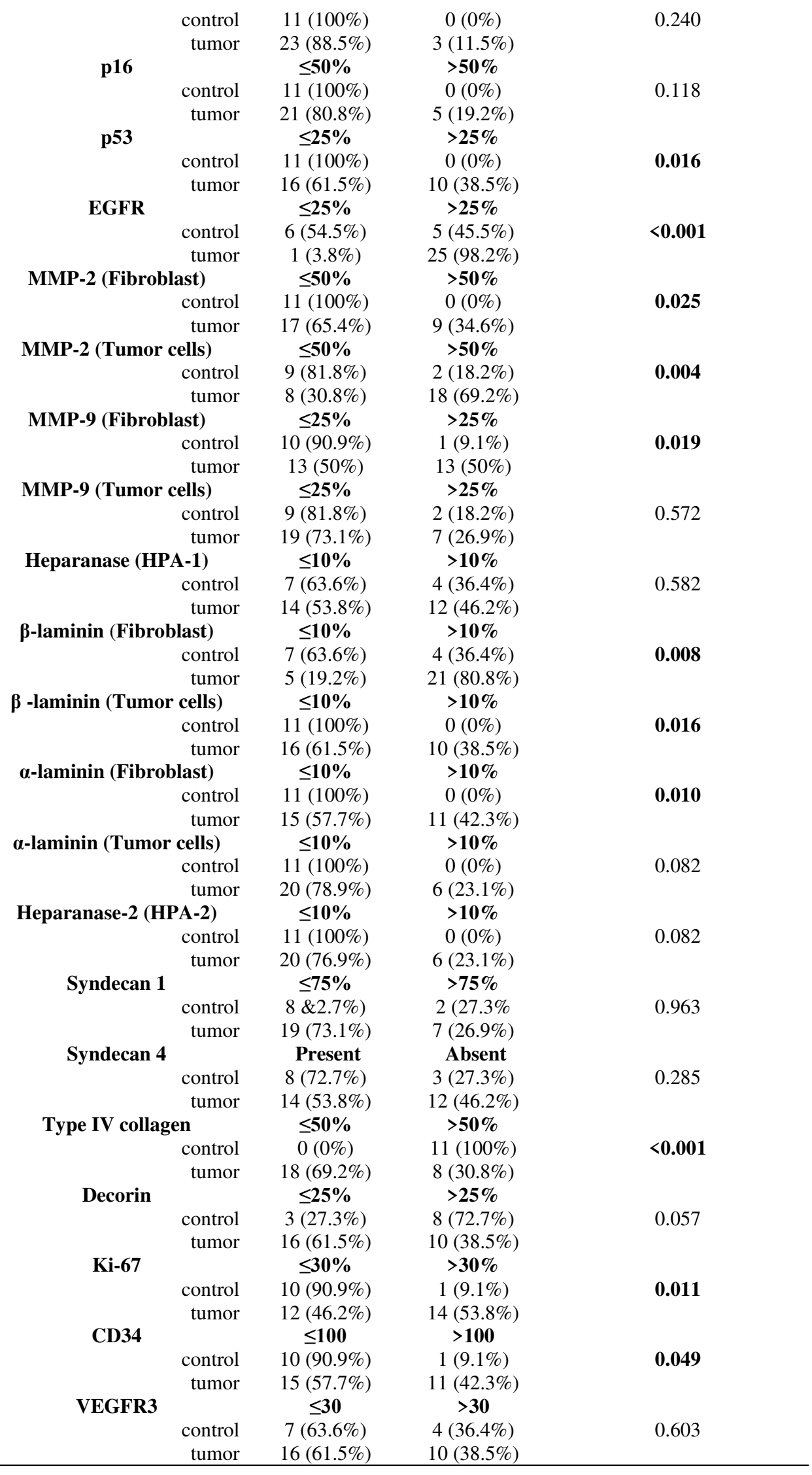


Comparison between differential microRNA expression and expression profile of immunohistochemical markers in SCC of the oral cavity

The microRNA expression levels were compared to various immunohistochemical markers that were classified based on staining intensity (high or low). There were several significant associations, and only these are shown in Table 4, demonstrating the interaction between microRNAs and various biological events in oral cavity SCC: significantly higher expression levels of let-7d-5p and miR17-5p, an increase in $\mathrm{Bcl}-2$, lower expression of miR-378a-3p and higher expression of MMP-2 in peritumoral fibroblasts; higher expression of miR-29a-3p and higher MMP-2 levels in tumor cells; higher expression of miR-485-5p and higher p53 levels, higher expression of miR-200b-3p and increased rates of laminin beta in tumor-associated fibroblasts; higher expression of miR-29a-3p and higher rates of laminin beta in neoplastic cells; higher expression of miR-376c-3p accompanied by more heparanase 2 in tumor cells; higher expression of miR-155-5p and higher rates of laminin alpha in tumor-related fibroblasts; higher miR-494-3p expression and lower continuity of type IV collagen in the basement membrane; and higher miR-203a-3p expression and higher decorin rates, higher miR27a-3p expression and lower miR-9-5p expression with higher Ki-67 levels, higher miR99a-5p expression in high CD34-density microvessels and lower expression of miR-221$3 p$ associated with a higher density of microvessels stained with VEGFR3.

Table 4. Significant associations between various microRNAs and immunohistochemical markers.

\begin{tabular}{lccccc}
\hline \multicolumn{7}{c}{ microRNA } & \multicolumn{7}{c}{ IMMUNOHISTOCHEMICAL MARKERS } & p (Mann-Whitney) \\
\hline \multicolumn{7}{c}{ Bcl-2 } \\
miR-let-7d-5p* & -5.162 & 0.179 & -3.569 & 0.232 & \\
miR-1-3p & -0.793 & 0.241 & 2.040 & 0.000 & 0.005 \\
miR-106b-5p & -3.759 & 0.242 & -1.203 & 0.424 & 0.007 \\
miR-126-3p & -1.190 & 0.161 & 0.000 & 0.419 & 0.012 \\
miR-16-5p & -4.268 & 0.194 & -2.588 & 0.431 & 0.032 \\
miR-17-5p* & -5.293 & 0.262 & -2.777 & 0.785 & 0.018 \\
miR-221-3p & -2.339 & 0.205 & -1.258 & 0.370 & 0.011 \\
miR-222-3p & -5.638 & 0.252 & -3.383 & 0.201 & 0.027 \\
miR-27a-3p & -2.108 & 0.171 & -0.705 & 0.230 & 0.016 \\
miR-376c-3p & -7.154 & 0.312 & -3.214 & 0.952 & 0.003 \\
miR-99a-5p & -2.535 & 0.181 & -1.541 & 0.329 & 0.004 \\
& \multicolumn{7}{c}{ MMP-2 (fibroblasts) } \\
miR-let-7b-5p & -1.518 & 0.199 & -2.289 & $>50 \%$ & 0.041 \\
miR-1-3p & -0.439 & 0.302 & -1.235 & 0.417 & 0.039 \\
& \multicolumn{7}{c}{$50 \%$}
\end{tabular}




\begin{tabular}{|c|c|c|c|c|c|}
\hline miR-133a-3p & -1.205 & 0.373 & -5.928 & 1.329 & 0.001 \\
\hline miR-192-5p & -4.085 & 0.559 & -5.953 & 0.754 & 0.028 \\
\hline miR-375 & -0.674 & 0.215 & -1.449 & 0.233 & 0.013 \\
\hline miR-378a-3p* & -2.205 & 0.281 & -4.630 & 0.813 & 0.016 \\
\hline miR-485-5p & -4.487 & 0.421 & -6.711 & 0.795 & 0.039 \\
\hline \multicolumn{6}{|c|}{ MMP-2 (lesion) } \\
\hline & \multicolumn{2}{|c|}{$</=50 \%$} & \multicolumn{2}{|c|}{$>50 \%$} & \\
\hline \multirow[t]{2}{*}{ miR-29a-3p } & -3.166 & 0.336 & $\begin{array}{l}-2.633 \\
\text { p53 }\end{array}$ & 0.150 & 0.047 \\
\hline & \multicolumn{2}{|c|}{$<l=25 \%$} & \multicolumn{2}{|c|}{$>25 \%$} & \\
\hline miR-485-5p & -5.478 & 0.590 & -4.805 & 0.596 & 0.036 \\
\hline \multicolumn{6}{|c|}{ Laminin beta (fibroblasts) } \\
\hline & \multicolumn{2}{|c|}{$</=50 \%$} & \multicolumn{2}{|c|}{$>50 \%$} & 0.012 \\
\hline miR-200b-3p & -1.123 & 0.273 & -0.174 & 0.277 & \\
\hline \multicolumn{6}{|c|}{ Laminin beta (lesion) } \\
\hline & \multicolumn{2}{|c|}{$</=10 \%$} & \multicolumn{2}{|c|}{$>10 \%$} & \\
\hline miR-29a-3p & -3.070 & 0.210 & -2.340 & 0.223 & 0.008 \\
\hline \multicolumn{6}{|c|}{ Heparinase 2 (lesion) } \\
\hline & \multicolumn{2}{|c|}{$</=10 \%$} & \multicolumn{2}{|c|}{$>10 \%$} & \\
\hline $\operatorname{miR-376c-3p*~}$ & -7.255 & 0.307 & -3.667 & 0.839 & 0.001 \\
\hline miR-378a-3p & -3.145 & 0.332 & -1.147 & 0.946 & 0.013 \\
\hline \multicolumn{6}{|c|}{ Laminin alpha (fibroblasts) } \\
\hline & \multicolumn{2}{|c|}{$</=10 \%$} & \multicolumn{2}{|c|}{$>10 \%$} & \\
\hline miR-155-5p* & -7.108 & 0.377 & -5.514 & 0.421 & 0.006 \\
\hline miR-16-5p & -4.451 & 0.218 & -3.393 & 0.325 & 0.032 \\
\hline miR-17-5p & -5.441 & 0.349 & -4.228 & 0.360 & 0.011 \\
\hline $\operatorname{miR}-200 c-3 p$ & -1.897 & 0.138 & -0.955 & 0.374 & 0.018 \\
\hline \multicolumn{6}{|c|}{ Decorin } \\
\hline & \multicolumn{2}{|c|}{$</=25 \%$} & & & \\
\hline miR-203a-3p & -3.449 & 0.358 & -2.621 & 0.257 & 0.031 \\
\hline & & Typ & IV collag & & \\
\hline & & & & & \\
\hline miR-494-3p & -3.287 & 0.729 & -5.927 & 0.527 & 0.042 \\
\hline & & & Ki-67 & & \\
\hline & & & & & \\
\hline miR-16-5p & -4.512 & 0.258 & -3.590 & 0.251 & 0.017 \\
\hline miR-17-5p & -5.593 & 0.310 & -4.190 & 0.438 & 0.002 \\
\hline miR-200a-3p & -3.059 & 0.224 & -2.182 & 0.241 & 0.013 \\
\hline miR-27a-3p* & -2.267 & 0.215 & -1.616 & 0.255 & 0.044 \\
\hline $\operatorname{miR}-376 c-3 p$ & -7.459 & 0.478 & -5.839 & 0.545 & 0.046 \\
\hline miR-9-5p* & -1.503 & 0.384 & -2.659 & 0.351 & 0.010 \\
\hline & & & CD34 & & \\
\hline miR-106b-5p & -4.145 & 0.309 & -2.630 & 0.293 & 0.022 \\
\hline miR-125 & -1.089 & 0.146 & -0.635 & 0.224 & 0.023 \\
\hline miR-99a-5p* & -2.602 & 0.216 & -2.149 & 0.283 & 0.015 \\
\hline & & & EGFR3 & & \\
\hline & & & & & \\
\hline miR-125b-5p & -0.358 & 0.165 & -0.765 & 0.253 & 0.014 \\
\hline miR-143-3p & -0.641 & 0.215 & -1.224 & 0.174 & 0.017 \\
\hline $\operatorname{miR}-221-3 p *$ & -1.992 & 0.214 & -2.703 & 0.366 & 0.020 \\
\hline miR-27a-3p & -1.863 & 0.253 & -2.200 & 0.165 & 0.031 \\
\hline miR-29c-3p & -2.347 & 0.217 & -2.761 & 0.154 & 0.047 \\
\hline miR-99a-5p & -2.325 & 0.196 & -2.668 & 0.328 & 0.027 \\
\hline
\end{tabular}

* miRs differentially expressed according to multivariate linear regression analysis (miR values presented as the mean and standard error) 


\section{Discussion}

The possibility of predicting cancer prognosis could bring great benefits to patients, allowing the selection and magnitude of therapy. MicroRNAs have made this possibility real, being highly promising as biomarkers of prognosis and targets of therapies while also being fundamental in tumor progression and influencing cell survival, differentiation, apoptosis, and migration, with several studies showing their association with oncogenesis and tumor infiltration $(30,31)$. The detailed analysis of these molecules and their association with changes in the extracellular matrix is essential for neoplasia infiltration, especially in the SCC of the oral cavity $(9,10,32)$.

The remodeling of the extracellular matrix is fundamental for the invasion and migration of neoplastic cells, and cancer-associated fibroblasts (CAFs) play an important role in the process, for example, through the synthesis of MMPs and laminins, as demonstrated in our study, in which the oral cavity SCC group showed higher expression of MMPs and laminins than the control group. Metalloproteinases, especially MMP-2 and MMP-9, from the gelatinase class degrade type IV collagen, present in the basement membrane, whose rupture is a watershed moment in the phenotypic transformation to malignancy, as demonstrated in our study by means of immunohistochemical analysis of type IV collagen and its loss of continuity in the SCC group. A study by Agarwal et al. (33) demonstrated lower immunohistochemical expression of type IV collagen directly proportional to the loss of oral cavity SCC differentiation. With the progression of invasion and the development of a tumor microenvironment, the synthesis of MMPs opens passageways through type I collagen, allowing cancer to gain territory $(34,35)$. A study by Ba et al. (36) evaluated the difference in the biological behavior of CAFs and 
peritumoral fibroblasts, and the expression of MMP-2, analyzed by qRT-PCR and ELISA, was higher in the CAF group than in the peritumoral fibroblast group.

The involvement of microRNAs as regulatory mechanisms for ECM changes is being increasingly elucidated, and one of the theories involves paracrine interactions between the tumor and tumor microenvironment cells, such as fibroblasts, involving enveloped microRNAs sent in 30-100 $\mathrm{nm}$ vesicles, called exosomes (37). As demonstrated by Baroni et al. (38), triple-negative mammary carcinoma tissues transform neighboring fibroblasts and fibroblasts associated with cancer through the transport of microRNA by exosomes, with these lesions having lower E-cadherin expression and a greater possibility of migration. Our study showed higher miR-21-5p and lower miR-1$3 p$ expression when there was greater immunoreactivity for MMP-2 in fibroblasts associated with the tumor, as well as greater vascular proliferation, as demonstrated by immunoreactivity to CD34 when there was greater miR-133-3p expression, despite other also detected associations.

The findings of the present study are vast and should be further explored. As limitations, we note that the sample size used was small, even for an experimental model. We believe that microRNAs identified as differentially expressed between superficial and thick tumors should be validated in larger cohorts. However, the study was pioneer in this field and it is one of the biggest in number of microRNAs evaluated. Moreover, it was the first one to demonstrate the association of microRNAs in the ECM remodeling in oral cavity SCC patients.

In the present study, high miR-21-5p and miR-106-5p expression and low miR320a and miR-222-3p expression were predictive of malignancy. Individually, the high expression of miR-21-5p showed the highest accuracy in the differentiation of tumor specimens from the healthy mucosa of the mouth. In addition, there was greater p53, 
EGFR, MMP-2, laminin beta, Ki-67 and CD34 expression in tumor cells than in healthy mucosal epithelial cells, increased MMP-2, MMP-9, laminin alpha and laminin beta expression in tumor-related fibroblasts compared to fibroblasts of the extracellular matrix of the healthy oral mucosa, and lower continuity of type IV collagen in the basal membrane of tumor specimens. These results demonstrate the biological effects of microRNAs on the carcinogenesis of SCC of the oral cavity as well as the intense modification of the tumor microenvironment. 


\section{References}

1. Ferlay J, Shin H-R, Bray F, Foreman D, Mathers C, Parkin DM. GLOBOCAN 2008 v1.2, Cancer Incidence and Mortality Worldwide: IARC CancerBase No. 10 [Internet]. International Agency for Research on Cancer. 2010.

2. World Health Organization. Global Health Observatory. Geneva: World Health Organization; 2018. who.int/gho/database/en/. Accessed November 11 2019. No Title.

3. Alvarenga LDM, Ruiz MT, Pavarino-Bertelli ÉC, Ruback MJC, Maniglia JV, Goloni-Bertollo EM. Avaliação epidemiológica de pacientes com câncer de cabeça e pescoço em um hospital universitário do noroeste do estado de São Paulo. Braz J Otorhinolaryngol. 2008;74(1):68-73.

4. Cooper JS, Porter K, Mallin K, Hoffman HT, Weber RS, Ang KK, et al. National cancer database report on cancer of the head and neck: 10-Year update. Head Neck. 2009;31(6):748-58.

5. De Camargo Cancela M, Voti L, Guerra-Yi M, Chapuis F, Mazuir M, Curado MP. Oral cavity cancer in developed and in developing countries: Populationbased incidence. Head Neck. 2010;32(3):357-67.

6. Radoï L, Menvielle G, Cyr D, Lapôtre-Ledoux B, Stücker I, Luce D, et al. Population attributable risks of oral cavity cancer to behavioral and medical risk factors in France: Results of a large population-based case-control study, the ICARE study. BMC Cancer. 2015;15:827.

7. de Matos LL, Miranda GA, Cernea CR. Prevalence of oral and oropharyngeal human papillomavirus infection in Brazilian population studies: A systematic 
review. Brazilian Journal of Otorhinolaryngology. 2015. p. 81(5):554-67.

8. Takes RP, Rinaldo A, Silver CE, Piccirillo JF, Haigentz M, Suárez C, et al. Future of the TNM classification and staging system in head and neck cancer. Head and Neck. 2010. p. 32(12):1693-711.

9. Matos LL De, Manfro G, Santos RV Dos, Stabenow E, Mello ES De, Alves VAF, et al. Tumor thickness as a predictive factor of lymph node metastasis and disease recurrence in $\mathrm{T} 1 \mathrm{~N} 0$ and $\mathrm{T} 2 \mathrm{~N} 0$ squamous cell carcinoma of the oral tongue. Oral Surg Oral Med Oral Pathol Oral Radiol. 2014;118(2):209-17.

10. Pentenero M, Gandolfo S, Carrozzo M. Importance of tumor thickness and depth of invasion in nodal involvement and prognosis of oral squamous cell carcinoma: A review of the literature. Head and Neck. 2005. p. 27(12):1080-91.

11. Moore C, Kuhns JG, Greenberg RA. Thickness as Prognostic Aid in Upper Aerodigestive Tract Cancer. Arch Surg. 1986;121(12):1410-4.

12. d'Alessandro AF, Pinto FR, Lin CS, Kulcsar MAV, Cernea CR, Brandão LG, et al. Oral cavity squamous cell carcinoma: Factors related to occult lymph node metastasis. Braz J Otorhinolaryngol. 2015;81(3):248-54.

13. Lin CS, de Oliveira Santos AB, Silva EL e., de Matos LL, Moyses RA, Kulcsar MAV, et al. Tumor volume as an independent predictive factor of worse survival in patients with oral cavity squamous cell carcinoma. Head Neck. 2017;39(5):960-4.

14. Li X, Wu Z, Fu X, Han W. A microRNA component of the neoplastic microenvironment: Microregulators with far-reaching impact. BioMed Research International. 2013. p. 762183.

15. Hanahan D, Weinberg RA. Hallmarks of cancer: The next generation. Cell. 2011. p. $144(5): 646-74$. 
16. Rodrigues LMR, Theodoro TR, Matos LL, Mader AM, Milani C, da Silva Pinhal MA. Heparanase isoform expression and extracellular matrix remodeling in intervertebral disc degenerative disease. Clinics. 2011;66(5):903-9.

17. Matos LL, Suarez ER, Theodoro TR, Trufelli DC, Melo CM, Garcia LF, et al. The profile of heparanase expression distinguishes differentiated thyroid carcinoma from benign neoplasms. PLoS One. 2015;10(10):e0141139.

18. Wentz-Hunter KK, Potashkin JA. The Role of miRNAs as Key Regulators in the Neoplastic Microenvironment. Mol Biol Int. 2011;839872.

19. Lopes CC, Dietrich CP, Nader HB. Specific structural features of syndecans and heparan sulfate chains are needed for cell signaling. Brazilian J Med Biol Res. 2006;39(2):157-67.

20. Bernfield M, Götte M, Park PW, Reizes O, Fitzgerald ML, Lincecum J, et al. Functions of Cell Surface Heparan Sulfate Proteoglycans. Annu Rev Biochem. 1999;68:729-77.

21. Piccinini AM, Midwood KS. Illustrating the interplay between the extracellular matrix and microRNAs. Int J Exp Pathol. 2014;95(3):158-80.

22. Fang Z, Rajewsky N. The impact of miRNA target sites in coding sequences and in 3'UTRs. PLoS One. 2011;6(3):e18067.

23. Calin GA, Sevignani C, Dumitru CD, Hyslop T, Noch E, Yendamuri S, et al. Human microRNA genes are frequently located at fragile sites and genomic regions involved in cancers. Proc Natl Acad Sci U S A. 2004;101(9):2999-3004.

24. John K, Wu J, Lee BW, Farah CS. MicroRNAs in head and neck cancer. International Journal of Dentistry. 2013. p. 2013:650218.

25. Gorenchtein M, Poh CF, Saini R, Garnis C. MicroRNAs in an oral cancer context-from basic biology to clinical utility. Journal of Dental Research. 2012. 
p. 91(5):440-6.

26. Jia LF, Wei SB, Mitchelson K, Gao Y, Zheng YF, Meng Z, et al. MiR-34a

inhibits migration and invasion of tongue squamous cell carcinoma via targeting MMP9 and MMP14. PLoS One. 2014;9(9):e108435.

27. Knutsvik G, Stefansson IM, Aziz S, Arnes J, Eide J, Collett K, et al. Evaluation of Ki67 expression across distinct categories of breast cancer specimens: A Population-based study of matched surgical specimens, core needle biopsies and tissue microarrays. PLoS One. 2014;11(3):e0150979.

28. Nassif AE, Filho RT. Immunohistochemistry expression of tumor markers CD34 and P27 as a prognostic factor of clinically localized prostate adenocarcinoma after radical prostatectomy. Rev Col Bras Cir. 2010;37(5):338-44.

29. WebMEV [Internet]. [cited $2020 \mathrm{Feb} 15]$. Available from: http://mev.tm4.org

30. Brockhoff HC, Kim RY, Braun TM, Skouteris C, Helman JI, Ward BB. Correlating the depth of invasion at specific anatomic locations with the risk for regional metastatic disease to lymph nodes in the neck for oral squamous cell carcinoma. Head Neck. 2017;39(5):974-9.

31. Yates LA, Norbury CJ, Gilbert RJC. The long and short of microRNA. Cell. 2013. p. 153:516-9.

32. Pinto FR, De Matos LL, Palermo FC, Kulcsar MAV, Cavalheiro BG, De Mello ES, et al. Tumor thickness as an independent risk factor of early recurrence in oral cavity squamous cell carcinoma. Eur Arch Oto-Rhino-Laryngology. 2014;271(6):1747-54.

33. Agarwal P, Ballabh R. Expression of type IV collagen in different histological grades of oral squamous cell carcinoma: An immunohistochemical study. J 
Cancer Res Ther. 2013;9(2):272-5.

34. Ribeiro RIMDA, Borges Jr. PC, Cardoso SV, Candelori I, Espíndola FS, Cassali GD, et al. Expression of matrix metalloproteinasis and their tissue inhibitors in basal cell carcinoma | Expressão de metaloproteinases de matriz e de seus inibidores teciduais em carcinomas basocelulares. J Bras Patol e Med Lab. $2008 ; 44(2): 115-21$.

35. Glentis A, Oertle P, Mariani P, Chikina A, El Marjou F, Attieh Y, et al. Cancerassociated fibroblasts induce metalloprotease-independent cancer cell invasion of the basement membrane. Nat Commun. 2017;8:924.

36. Ba P, Zhang X, Yu M, Li L, Duan X, Wang M, et al. Cancer associated fibroblasts are distinguishable from peri-tumor fibroblasts by biological characteristics in TSCC. Oncol Lett. 2019;18:2484-90.

37. Yang F, Ning Z, Ma L, Liu W, Shao C, Shu Y, et al. Exosomal miRNAs and miRNA dysregulation in cancer-associated fibroblasts. Molecular Cancer. 2017. p. 16:148.

38. Baroni S, Romero-Cordoba S, Plantamura I, Dugo M, D'Ippolito E, Cataldo A, et al. Exosome-mediated delivery of miR-9 induces cancer-Associated fibroblastlike properties in human breast fibroblasts. Cell Death Dis. 2016;7(7):e2312. 
Role de the funding source

Fundação de Amparo à Pesquisa do Estado de São Paulo (FAPESP - process 2016/01740-6)

Public funding to the research of the state of São Paulo - Brazil 


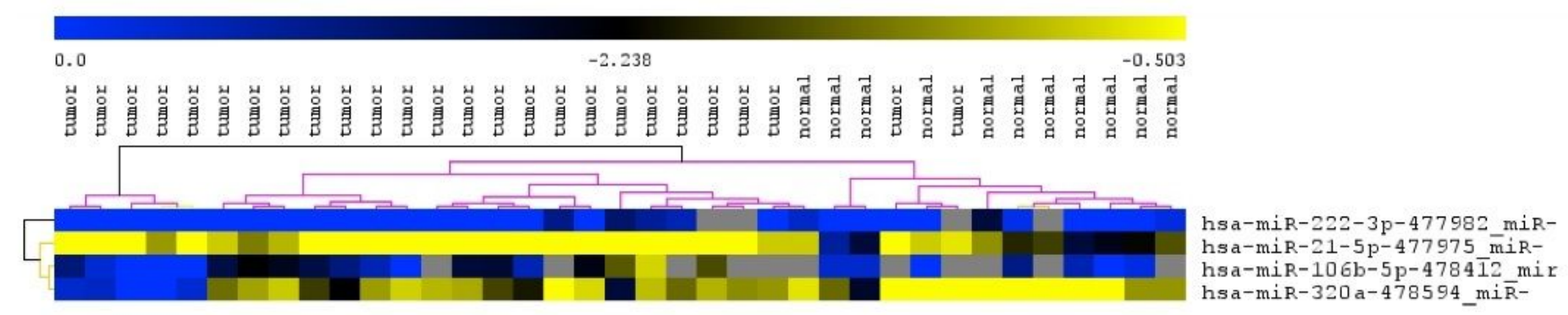

Figure 1

Heatmap showing the differentially expressed microRNAs and hierarchical cluster analysis of the samples for the differentiation of tumor specimens from healthy mucosa, considering only the microRNAs differentially expressed in the multivariate analysis (miR -21-5p, miR320a, miR-106b-5p and miR-222-3p). Yellow areas represent samples with lower expression, and blue areas represent samples with higher microRNA expression. 


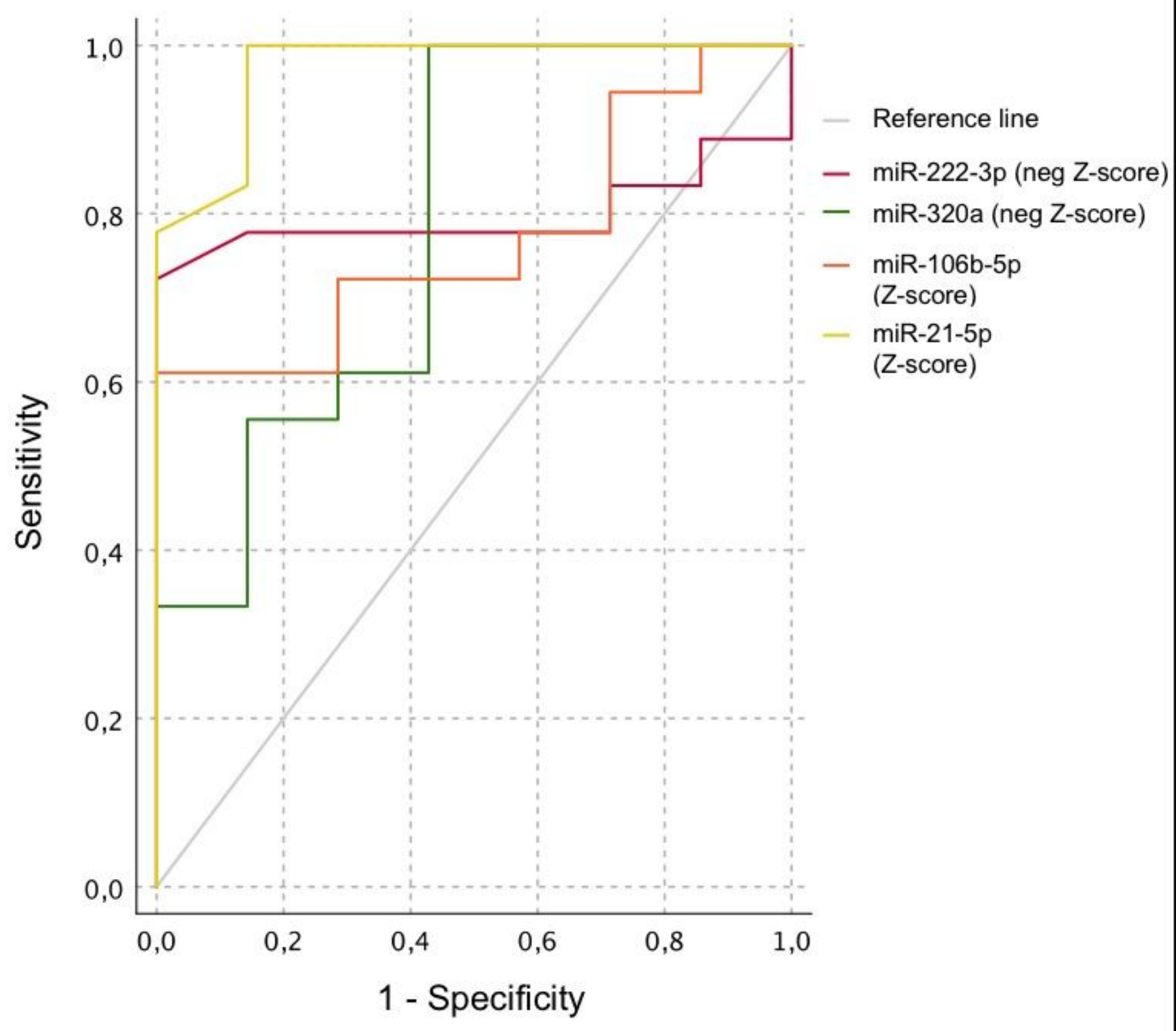

\section{Figure 2}

ROC (receiver operating characteristic) curve demonstrating the accuracy of miRNAs $21-5 p, 106 b-5 p, 222-$ $3 p$ and $320 a$ for the differential diagnosis of squamous cell carcinoma of the oral cavity from healthy mucosa. 